\title{
Macrobenthos Community in the Littoral Zone Water Area of Iboih Beach Sabang, Aceh
}

\author{
Rita Oktavia ${ }^{1}$, Nurdian Amin ${ }^{2}$ \\ ${ }^{1}$ Biology Education Study Program in College of STKIP Bina Bangsa Meulaboh \\ ${ }^{2}$ Biology Education Study Program at the Faculty of Tarbiyah and Teacher Training in Islamic State University \\ of Ar-Raniry \\ ritaoktavia87@gmail.com
}

\begin{abstract}
The aim of this study is to know the macrobenthos community in the littoral zone water area of Iboih beach, Aceh. Sample collection is conducted by using plot size of $1 \times 1$ meter, destructive sampling and non-destructive sampling methods. The results shows that macrobenthos inventory on Rubiah Island in Sabang, Aceh with destructive sampling methods is amounted to 8 species from 5 families, while Benthos obtained from non-destructive sampling methods is amounted to 8 species from 6 families. Macrobenthos inventory on the water of natural tourism area of Iboih beach Sabang. Diversity index is moderate with $H^{\prime}=$ 1.22061, and non-destructive value $H^{\prime}=1.31391$. Includes the category of moderate diversity. In Payau waters with destructive $H^{\prime}=1.74816$, nondestructive with a value of $H^{\prime}=1.7104$. Has a moderate diversity index. Macroentent inventory of the Teupin Layeu Mangrove Waters of Sabang City found 27 and 29 species of benthos. Among them (Meretrik meretrik) is amounted to 44, (Anadara cunearca) is amounted to 36 and many other species. With species diversity $H^{\prime}=3,2114$ indicates a high level of species diversity.
\end{abstract}

Keywords: Macrobenthos; Litoral zone water; Iboih beach.

\section{Introduction}

Aceh has a wealth of macrobenthos and macrobenthos aquatic organisms. Some of which have been surveyed include the white sand waters of Lhok Mee Aceh Besar found in various invertebrates, that is Gastropod Class, Holothuroidea, Sea urchin (Diadema, Sp), Chiton, sp. Ophiuroide (Oktavia, R. 2018).

Weh Island or Sabang City in the province of Aceh is one of the marine tourism destinations located at coordinates $95^{\circ} 14^{\prime} 11,39^{\prime \prime}$ BT and $05^{\circ} 53^{\prime}$ '38, 66" LU with an area of $153 \mathrm{~km}^{2}$. In this Weh Island have several other small islands that have biodiversity that has not been explored. Among Rubiah Island, Rondo Island and others.

Iboih Beach is a marine tourism area that stores a lot of natural wealth and diversity of living creatures that live in it. One of the aquatic biota in this beach is benthos. Benthos are organisms that live on the surface or in the basic sediments of a body of water. Benthos are all aquatic organisms that live on the basic substrate of a water, both sessile (attached) and vigil (moving freely).

Based on the place of life, benthos can be divided into epifauna and ifauna. Epifauna is benthos whose life is on the substrate of the water while infauna is benthos whose life is embedded in the substrate of the water base. (Barus T., 2004)

The role of benthos in a waters is to be able to recycle the organic material that is found or dissolved in a waters. In addition, benthos plays a role in helping the mineralization process and occupies an important position in the food chain and indicators of pollution of the aquatic environment.

Benthos as a basic aquatic organism that has a relatively fixed habitat. With these characteristics, changes in water quality and the substrate where it lives greatly affect the composition and abundance. The composition and abundance of macro invertebrates depends 
on their tolerance for environmental changes. Each community responds to changes in quality and habitat by adjusting to the community structure. (Effendi, 2003).

Benthos has a sensitivity to some pollutants, low mobility, easy to catch and has a long survival. Therefore, the role of benthos in the balance of an aquatic ecosystem can be an indicator of the current ecological conditions in a particular region. (Ajeng, Tri Purnami, et al., 2010). Apart from that, some or all life cycle of benthos is in the bottom of the waters, both sessile, creeping and digging holes. Therefore, the benthos research was carried out with the destructive or destructive method and the habitat without benthos (nondestructive) method. (Borror, 2000).

The main factors that influence the amount of benthos, species diversity, and dominance, among others, are the destruction of natural habitats, chemical pollution, and climate change (Ajeng Tri Purnami, et al., 2010). Biotic factors that influence are producers, which is a food source for benthos animals. The abiotic factors are the physics-chemistry of water which include temperature, current, dissolved oxygen (DO), biological oxygen demand (BOD) and chemistry (COD), as well as nitrogen $(\mathrm{N})$ content, water depth, and basic substrate. (Campbell, K. A, 2003).

\section{Research Methods}

This study was carried out on Weh Island, Sabang. The sampling was carried out in three different habitats, there are Rubiah Island, natural tourist area waters, and mangrove Teupin Layeu waters. Sampling was carried out in May-June 2018 and identification was continued at the Biology Laboratory of Islamic State University of Ar-Raniry Banda Aceh.

Sample collection was carried out using 1 x 1 meter plots, destructive sampling and nondestructive sampling methods. Benthos with destructive sampling method initially selected observation areas such as the sea littoral area. Observational plots were made with $1 \mathrm{x} 1$ meter measurement when low tide. Measured $\mathrm{pH}$, salinity, temperature and water depth. Passed down the ekmand grap slowly to the bottom of the waters, then released the pendulum. Then slowly lifted, put mud into the filter, then sifted, separated specimens from mud. Animal specimens were put into a sample bottle and given $4 \%$ formalin, identified and entered into the observation table. For shallow waters, it is done by scraping or dismantling benthos habitat. Furthermore, benthos is calculated using scratches or by hand directly in the square or that has been made.

Benthos with nondestructive sampling method selected observation areas such as the sea littoral area, made an observation plot with a size of $1 \times 1$ meter at low tide, measured $\mathrm{pH}$, salinity, temperature and water depth, calculated directly benthos that is in the square or that has been made, then identified and tabulated in the table.

There are three benthos sampling locations on Weh Island Sabang. The determination of the observation station is done by purposive sampling by determining the area identified by the existence of benthos. The distance of each station ranges from 10 meters and the selection of each sampling point based on the depth zone of the waters that is shallow water $( \pm 30 \mathrm{~cm})$, medium $( \pm 60 \mathrm{~cm})$ and deep $( \pm 90 \mathrm{~cm})$.

Unknown sample types are put into sample bottles which contains $70 \%$ alcohol for temporary preservation. Observation and identification of benthos were carried out in the Biology Education Study Program Laboratory of FTK Islamic State University of Ar-Raniry Banda Aceh.

Benthos diversity $\left(\mathrm{H}^{\prime}\right)$ is calculated using the Shannon-Winners Diversity Index formula as follows: 


\section{$\mathbf{H}^{\prime}=-\sum(\mathbf{P i})(\mathbf{L n} \mathrm{Pi})$ \\ Where $\mathbf{P i}=\frac{n i}{N}$}

The results obtained can then be categorized into 3 categories, namely:

1. If $\mathrm{H}^{\prime}<1$, the diversity index is categorized as Low.

2. If $\mathrm{H}^{\prime} 1<\mathrm{H}^{\prime}<3$, the diversity index is categorized as Medium.

3. If the results of $\mathrm{H}^{\prime}>3$ then the diversity index is categorized as High (Fachrul, 2006).

\section{Discussion}

Based on the results of the identification and analysis of the data, the benthos community on Rubiah Island, the waters of the natural tourism area, and the mangrove waters of Teupin Layeu were found as follows:

\subsection{Benthos Community on Rubiah Island}

The number of species found on the littoral of the Rubiah Islands in the Sukakarya SubSubdistrict of Sabang City is amounted to 16 species of benthos out of 11 families. Types of benthos found in the waters of Rubiah Islands in the Sukakarya SubSubdistrict of Sabang City were obtained by destructive sampling methods of 8 species from 5 families, including Crassostrea gigas amounted to 19, Saccostrea cucculata amounted to 5, Crassostrea sp amounted to 3, Paltella vulgata amounted to 3, Chiton sp. amounted to 2, Cerastoderma edule amounted to 3, Mytilus edulis amounted to 9, and Planaxis sulcatus amounted to 7.

Whereas Benthos which obtained from non-destructive sampling methods are amount to 8 species from 6 families including Perna viridis amounted to 1, Mytilus viridis amounted to 2, Meretrix meretrik amounted to 11, Paphia textile amounted to 3, Anadara (cunearca) amounted to 13, Scylla serrata amounted to 2, Cerithidae cingulata amounted to 8, and Stramonita haemastoma amounted to 9. Family and types of benthos found in Rubiah Island in Sukakarya SubSubdistrict, Sabang City as a whole can be seen in Table 1.

Table 1 Families, Species and Diversity Index of Benthos in the Waters of Rubiah Island in Sukakarya SubSubdistrict, Sabang City

\begin{tabular}{|c|c|c|c|}
\hline No. & Families & Species & Total \\
\hline \multirow[t]{3}{*}{1.} & Ostreidae & 1. Crassostrea gigas & 19 \\
\hline & Ostreidae & 2. Saccostrea cucculata & 5 \\
\hline & Ostreidae & 3. Crassostrea sp & 3 \\
\hline \multirow[t]{3}{*}{2.} & Mytilidae & 4. Perna viridis & 1 \\
\hline & Mytilidae & 5. Mytilus viridis & 2 \\
\hline & Mytilidae & 6. Mytilus edulis & 9 \\
\hline \multirow[t]{2}{*}{3.} & Veneridae & 7. Meretrix meretrik & 11 \\
\hline & Veneridae & 8. Paphia textile & 3 \\
\hline 4. & Arcidae & 9. Anadara (cunearca) & 13 \\
\hline 5. & Fortunidae & 10. Scylla serrata & 2 \\
\hline 6. & Potamididae & 11. Cerithidae cingulata & 8 \\
\hline 7. & Patelidae & 12. Paltella vulgate & 3 \\
\hline 8. & Chidondae & 13. Chiton $s p$ & 2 \\
\hline 9. & Muricidae & 14. Stramonita haemastoma & 9 \\
\hline
\end{tabular}




\begin{tabular}{llc} 
10. Cardiidae & 15. Cerastoderma edule & 3 \\
11. & Planaxidae & 7 \\
\hline Total & 16. Planaxis sulcatus & 100 \\
\hline Diversity index $\left(\mathrm{H}^{\prime}\right)=-\Sigma \mathrm{Pi}$ Ln $\mathrm{Pi}=-(-2,49655122)=2,49655122$ \\
\hline
\end{tabular}

The Shannon Winner diversity index of benthos species in the waters of Rubiah Island, Sukakarya SubSubdistrict, Sabang City is classified as moderate, which is indicated by the benthos diversity index of 2.49655122 .

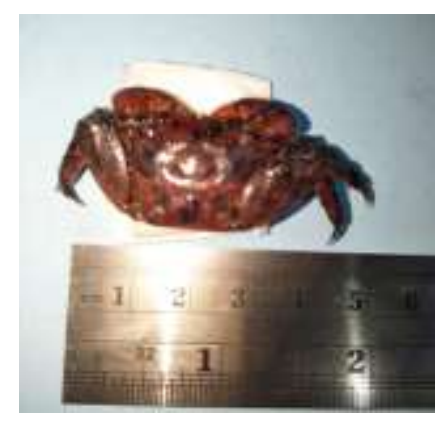

Scylla serrata

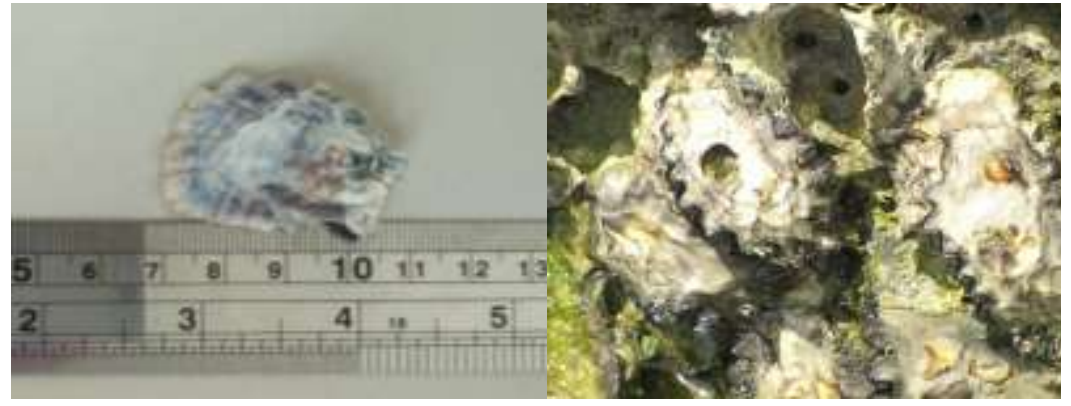

Crasostrea sp.

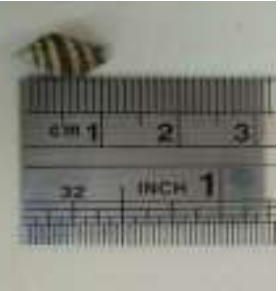

Planaxis sulcatus

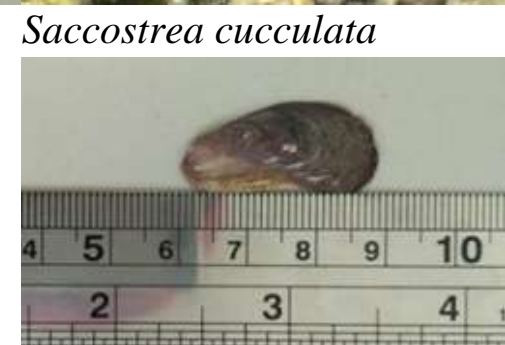

Mytilus edulis

\subsection{Benthos Community in the Waters of the Natural Tourism Area}

Benthos diversity in the Iboih coastal area with destructive sampling method is classified as moderate, with a diversity index value of $\mathrm{H}^{\prime}=1.22061$ (1.2). The most dominant species is Parathelphusa sp. from the Parathelphusidae family is amounted to 5 species and the least species found is Pilsbryoconcha exilis from the Parathelphusidae family which is only 1 species (Table 2). Whereas benthos sampling uses non-destructive sampling method. (Table 3.) found that the dominant species are 9 species of Mytilus edulis. The diversity index is classified as moderate with the value $\mathrm{H}^{\prime}=1.31391$ (1.3).

Inventory uses destructive sampling method in Payau waters, the type of benthos found is from 6 different families. The species that dominate are from the Arcidae family with the species Barbatia sp. is amounted to 3 species. The diversity index is classified as moderate with a value of $\mathrm{H}^{\prime}=1.74816$ (1.7) (Table 4).

Inventory uses a non-destructive sampling method in Payau waters in the natural tourism area of Iboih, Sabang (Table 5). That is amounted to 6 families with predominant species that are derived from the Isognomonidae family with Isognomon sp. Is amounted to 4 species. The diversity index is classified as moderate with the value $\mathrm{H}^{\prime}=1.7104$ (1.7). 
Table 2. Benthos Diversity in Waters of Iboih Beach in Destructive Sampling

\begin{tabular}{cllcc}
\hline No & Species & Families & Total & H' \\
\hline $\mathbf{1}$ & Cerithidea cingulata & Potamididae & 2 & 0,32189 \\
$\mathbf{2}$ & Pilsbryoconcha exilis & Unionidae & 1 & 0,23026 \\
$\mathbf{3}$ & Parathelphusa convexa & Parathelphusidae & 2 & 0,32189 \\
$\mathbf{4}$ & Parathelphusa sp. & Parathelphusidae & 5 & 0,34657 \\
\hline \multicolumn{2}{l}{ Total } & & 10 & 1,22061 \\
\hline
\end{tabular}

Diversity index $\left(\mathrm{H}^{\prime}\right)=-\sum \mathrm{Pi}$ Ln Pi $=-(-1.22061)=1,22061$

Table 3. Benthos Diversity in Waters of Iboih Beach in Non-Destructive Sampling

\begin{tabular}{cllcc}
\hline No & Species & Families & Total & $\mathbf{H}^{\prime}$ \\
\hline $\mathbf{1}$ & Littorina $s p$ & Littorinidae & 5 & 0,32679 \\
$\mathbf{2}$ & Cerastoderma edule & Cardiidae & 3 & 0,2599 \\
$\mathbf{3}$ & Mytilus edulis & Mytilidae & 9 & 0,36781 \\
$\mathbf{4}$ & Planaxis sulcatus & Planaxidae & 7 & 0,35937 \\
\hline \multicolumn{2}{l}{ Total } & & 24 & 1,31391 \\
\hline
\end{tabular}

Diversity index $\left(\mathrm{H}^{\prime}\right)=-\sum$ Pi Ln Pi $=-(-1,313911)=1,31391$

Table 4. Benthos Diversity in Waters of Payau in the Natural Tourism Area of Iboih in Destructive Sampling

\begin{tabular}{cllcc}
\hline No & Species & Families & Total & H'$^{\prime}$ \\
\hline $\mathbf{1}$ & Barbatia sp. & Arcidae & 3 & 0,34657 \\
$\mathbf{2}$ & Natica sp. & Naticidae & 2 & 0,29863 \\
$\mathbf{3}$ & Nerita sp. & Neritidae & 2 & 0,20708 \\
$\mathbf{4}$ & Scylla sp. & Portunidae & 1 & 0,29863 \\
$\mathbf{5}$ & Monodonta sp. & Trochidae & 2 & 0,29863 \\
$\mathbf{6}$ & Isognomon sp. & Isognomonidae & 2 & 0,29863 \\
\hline \multicolumn{2}{l}{ Total } & & 12 & 1,74816 \\
\hline
\end{tabular}

Diversity index $\left(\mathrm{H}^{\prime}\right)=-\Sigma(\mathrm{Pi})(\mathrm{InPi})=-(-1,74816)=1,74816$

Table 5. Benthos Diversity in Waters of Payau in the Natural Tourism Area of Iboih in NonDestructive Sampling

\begin{tabular}{|c|c|c|c|c|}
\hline No & Species & Families & Total & $\overline{\mathbf{H}^{\prime}}$ \\
\hline 1 & Nerita sp. & Neritidae & 1 & 0,1885 \\
\hline 2 & Telescopium telescopium & Potamididae & 2 & 0,2779 \\
\hline 3 & Monodonta sp. & Trochidae & 3 & 0,3301 \\
\hline 4 & Scylla sp. & Portunidae & 2 & 0,2779 \\
\hline 5 & Littorina scabra & Littorinidae & 2 & 0,2779 \\
\hline 6 & Isognomon sp. & Isognomonidae & 4 & 0,3579 \\
\hline \multicolumn{2}{|c|}{ Total } & & 12 & 1,7104 \\
\hline
\end{tabular}


Budapest International Research in Exact Sciences (BirEx) Journal

Volume 1, No 3, July 2019, Page: 106-114

e-ISSN: 2655-7827 (Online), p-ISSN: 2655-7835 (Print) www.bircu-journal.com/index.php/birex emails: birex.journal@gmail.com birex.journal.qa@gmail.com

\subsection{Benthos Community in the Teupin Layeu Manggrove Waters}

The results of iventarisation were obtained by 42 benthos species in the Teupin Layeu area of Sabang City by using destructive sampling methods and 42 types of benthos by using non destructive sampling methods.

Table 6. Benthos Diversity of Destructive Sampling in the Teupin Layeu Mangrove Waters of Sabang City

\begin{tabular}{|c|c|c|c|c|c|c|c|c|c|c|c|c|}
\hline $\begin{array}{l}\text { Po- } \\
\text { int }\end{array}$ & No. & Phylum & Class & Ordo & Families & Genus & Species & $\Sigma$ & $\begin{array}{c}\mathrm{Pi} \\
(\mathrm{NI} / \mathrm{n})\end{array}$ & Ln. Pi & Pi.Ln.PI & $\mathrm{H}^{\prime}$ \\
\hline \multirow[t]{3}{*}{1} & 1 & Mollusca & Bivalvia & $\begin{array}{l}\text { Poecilocle- } \\
\text { rina }\end{array}$ & $\begin{array}{l}\text { Isognomo- } \\
\text { nidae }\end{array}$ & Isognomon & $\begin{array}{l}\text { Isognomon } \\
\text { ephippium }\end{array}$ & 10 & 0,05128 & $-2,9705$ & $-0,1523308$ & $-0,1523308$ \\
\hline & 2 & Mollusca & Bivalvia & $\begin{array}{l}\text { Haplo- } \\
\text { scrindo }\end{array}$ & $\begin{array}{l}\text { Margaritife- } \\
\text { ridae }\end{array}$ & Margaritifera & $\begin{array}{l}\text { Margaritifera } \\
\text { margaritifera }\end{array}$ & 6 & 0,03077 & $-3,4812$ & $-0,1071142$ & $-0,1071142$ \\
\hline & 3 & Mollusca & Bivalvia & $\begin{array}{l}\text { Hipposngia- } \\
\text { dae }\end{array}$ & Corbiludae & Polymesoda & $\begin{array}{l}\text { Polymesoda } \\
\text { expansa }\end{array}$ & 1 & 0,00513 & $-5,2726$ & $-0,0270392$ & $-0,0270392$ \\
\hline \multirow[t]{4}{*}{2} & 1 & Porifera & $\begin{array}{l}\text { Demo- } \\
\text { spongiae }\end{array}$ & $\begin{array}{l}\text { Poecilocle- } \\
\text { rina }\end{array}$ & Microcionidae & Microciona & Microciona $s p$ & 17 & 0,08718 & -243978 & $-21269,877$ & $-21269,877$ \\
\hline & 2 & Porifera & $\begin{array}{l}\begin{array}{l}\text { Demo- } \\
\text { spongiae }\end{array} \\
\end{array}$ & $\begin{array}{l}\text { Haplo- } \\
\text { scrindo }\end{array}$ & Chalinidae & Haliclona & $\begin{array}{l}\text { Haliclona } \\
\text { oculata }\end{array}$ & 6 & 0,03077 & $-3,4812$ & $-0,1071142$ & $-0,1071142$ \\
\hline & 3 & Porifera & $\begin{array}{l}\begin{array}{l}\text { Demo- } \\
\text { spongiae }\end{array} \\
\end{array}$ & Keratosa & Hipposngiadae & Hipposngiae & $\begin{array}{l}\text { Hippospongia } \\
s p\end{array}$ & 13 & 0,06667 & $-2,708$ & $-0,1805333$ & $-0,1805333$ \\
\hline & 4 & Anidara & $\begin{array}{l}\text { Sclerac- } \\
\text { tinia }\end{array}$ & $\begin{array}{l}\text { Pocillopori- } \\
\text { dae }\end{array}$ & Pocilloporidae & Pocillopora & $\begin{array}{l}\text { Pocillopora } \\
\text { damicornis }\end{array}$ & 2 & 0,01026 & $-4,5795$ & $-0,0469692$ & $-0,0469692$ \\
\hline \multirow[t]{2}{*}{3} & 1 & Mollusca & Bivalvia & $\begin{array}{l}\text { Haplo- } \\
\text { scrindo }\end{array}$ & $\begin{array}{l}\text { Margaritiferida } \\
\text { e }\end{array}$ & Margaritifera & $\begin{array}{l}\text { Margaritifera } \\
\text { margaritifera }\end{array}$ & 3 & 0,01538 & $-4,1747$ & $-0,0642258$ & $-0,0642258$ \\
\hline & 2 & Porifera & $\begin{array}{l}\text { Gastro- } \\
\text { poda }\end{array}$ & $\begin{array}{l}\text { Neotaenioq- } \\
\text { lossa }\end{array}$ & Hydrobiidae & Pyrgulopsis & $\begin{array}{l}\text { Pyrgulopsis } \\
\text { nevadensis }\end{array}$ & 2 & 0,01026 & $-4,5795$ & $-0,0469692$ & $-0,0469692$ \\
\hline \multirow[t]{3}{*}{4} & 1 & Mollusca & Bivalvia & $\begin{array}{l}\text { Poecilocle- } \\
\text { rina }\end{array}$ & $e^{\text {Isognomonida }}$ & Isognomon & $\begin{array}{l}\text { Isognomon } \\
\text { ephippium }\end{array}$ & 10 & 0,05128 & $-2,9705$ & $-0,1523308$ & $-0,1523308$ \\
\hline & 2 & Mollusca & Bivalvia & $\begin{array}{l}\text { Haplo- } \\
\text { scrindo }\end{array}$ & Margaritiferida & Margaritifera & $\begin{array}{l}\text { Margaritifera } \\
\text { margaritifera }\end{array}$ & 6 & 0,03077 & $-3,4812$ & 0,1071142 & $-0,1071142$ \\
\hline & 3 & Mollusca & Bivalvia & $\begin{array}{l}\begin{array}{l}\text { Hippo- } \\
\text { sngiadae }\end{array} \\
\end{array}$ & Corbiludae & Polymesoda & $\begin{array}{l}\text { Polymesoda } \\
\text { expansa }\end{array}$ & 1 & 0,00513 & $-5,2726$ & $-0,0270392$ & $-0,0270392$ \\
\hline \multirow[t]{4}{*}{5} & 1 & Porifera & $\begin{array}{l}\text { Gastro- } \\
\text { poda }\end{array}$ & $\begin{array}{l}\text { Caeno- } \\
\text { gastropoda }\end{array}$ & Thiaridae & Tarebia & Pyrgulopsis & 5 & 0,02564 & $-3,6636$ & $-0,0939385$ & $-0,0939385$ \\
\hline & 2 & Porifera & $\begin{array}{l}\text { Gastro- } \\
\text { poda }\end{array}$ & $\begin{array}{l}\text { Caeno- } \\
\text { gastropoda }\end{array}$ & Hydrobiidae & Tryonia & \begin{tabular}{|l} 
Tryonia \\
clathrata
\end{tabular} & 1 & 0,00513 & $-5,2726$ & $-0,0270392$ & $-0,0270392$ \\
\hline & 3 & Porifera & $\begin{array}{l}\text { Gastro- } \\
\text { poda }\end{array}$ & $\begin{array}{l}\text { Caeno- } \\
\text { gastropoda }\end{array}$ & Lymnaeinae & Lymneas & $\begin{array}{l}\text { Lymneas } \\
\text { stagnalis }\end{array}$ & 2 & 0,01026 & $-4,5795$ & $-0,0469692$ & $-0,0469692$ \\
\hline & 4 & Porifera & $\begin{array}{l}\text { Gastro- } \\
\text { poda }\end{array}$ & $\begin{array}{l}\text { Neotaenioq- } \\
\text { lossa }\end{array}$ & Hydrobiidae & Pyrgulopsis & $\begin{array}{l}\text { Pyrgulopsis } \\
\text { nevadensis }\end{array}$ & 5 & 0,02564 & $-3,6636$ & $-0,0939385$ & $-0,0939385$ \\
\hline \multirow[t]{5}{*}{6} & 1 & Mollusca & $\begin{array}{l}\text { Gastro- } \\
\text { poda }\end{array}$ & $\begin{array}{l}\text { Megastro- } \\
\text { poda }\end{array}$ & Littorinidae & Littoraria & $\begin{array}{l}\text { Lttoraria } \\
\text { scabra }\end{array}$ & 18 & 0,09231 & $-2,3826$ & $-0,2199323$ & $-0,2199323$ \\
\hline & 2 & Mollusca & $\begin{array}{l}\text { Gastro- } \\
\text { poda }\end{array}$ & & Thiaridae & Thiara & $\begin{array}{l}\text { Thiara } \\
\text { cancellata }\end{array}$ & 1 & 0,00513 & $-5,2726$ & $-0,0270392$ & $-0,0270392$ \\
\hline & 3 & Mollusca & $\begin{array}{l}\text { Gastro- } \\
\text { poda }\end{array}$ & $\begin{array}{l}\text { Eupulmo- } \\
\text { nata }\end{array}$ & Ellobidae & Ellobium & \begin{tabular}{|l|} 
Ellobium \\
aurimisdae
\end{tabular} & 3 & 0,01538 & $-4,1747$ & $-0,0642258$ & $-0,0642258$ \\
\hline & 4 & Atropoda & $\begin{array}{l}\text { Crusta- } \\
\text { ceae }\end{array}$ & Decapoda & Portunidae & Scylla & Scylla serrata & 2 & 0,01026 & $-4,5795$ & $-0,0469692$ & $-0,0469692$ \\
\hline & 5 & Mollusca & $\begin{array}{l}\text { Gastro- } \\
\text { poda }\end{array}$ & $\begin{array}{l}\text { Caeno- } \\
\text { gastropoda }\end{array}$ & Potamididae & Terebralia & $\begin{array}{l}\text { Terebralia } \\
\text { sulcata }\end{array}$ & 2 & 0,01026 & $-4,5795$ & $-0,0469692$ & $-0,0469692$ \\
\hline \multirow[t]{6}{*}{7} & 1 & Mollusca & Bivalvia & $\begin{array}{l}\text { Pteriomor- } \\
\text { phia }\end{array}$ & Arcoidae & Anadara & $\begin{array}{l}\text { Anadara } \\
\text { granosa }\end{array}$ & 1 & 0,00513 & $-5,2726$ & $-0,0270392$ & $-0,0270392$ \\
\hline & 2 & Mollusca & $\begin{array}{l}\text { Gastro- } \\
\text { poda }\end{array}$ & $\begin{array}{l}\text { Vetigastro- } \\
\text { poda }\end{array}$ & Ovinotidae & Ovinotis & Ovinotis ovina & 1 & 0,00513 & $-5,2726$ & $-0,0270392$ & $-0,0270392$ \\
\hline & 3 & Mollusca & $\begin{array}{l}\text { Gastro- } \\
\text { poda }\end{array}$ & $\begin{array}{l}\text { Neotaenioq- } \\
\text { lossa }\end{array}$ & Hydrobiidae & Pyrgulopsis & $\begin{array}{l}\text { Prygulopsis } \\
\text { nevadensis }\end{array}$ & 1 & 0,00513 & $-5,2726$ & $-0,0270392$ & $-0,0270392$ \\
\hline & 4 & Mollusca & $\begin{array}{l}\text { Gastro- } \\
\text { poda }\end{array}$ & $\begin{array}{l}\text { Caeno- } \\
\text { gastropoda }\end{array}$ & Thiaridae & Tarebia & \begin{tabular}{|l|} 
Tarebia \\
granifera
\end{tabular} & 1 & 0,00513 & $-5,2726$ & $-0,0270392$ & $-0,0270392$ \\
\hline & 5 & Mollusca & $\begin{array}{l}\text { Gastro- } \\
\text { poda }\end{array}$ & $\begin{array}{l}\text { Neriti- } \\
\text { morpha }\end{array}$ & Neritidae & Neritina & $\begin{array}{l}\text { Neritina } \\
\text { natalensis }\end{array}$ & 1 & 0,00513 & \begin{tabular}{|l|}
$-5,2726$ \\
\end{tabular} & $-0,0270392$ & $-0,0270392$ \\
\hline & 6 & Crutascea & $\begin{array}{l}\text { Malacostr } \\
\text { aca }\end{array}$ & Decapoda & Portunidae & Scylla & Scylla serrata & 1 & 0,00513 & $-5,2726$ & $-0,0270392$ & $-0,0270392$ \\
\hline
\end{tabular}


Budapest International Research in Exact Sciences (BirEx) Journal

Volume 1, No 3, July 2019, Page: 106-114

e-ISSN: 2655-7827 (Online), p-ISSN: 2655-7835 (Print) www.bircu-journal.com/index.php/birex emails: birex.journal@gmail.com birex.journal.qa@gmail.com

\begin{tabular}{|c|c|c|c|c|c|c|c|c|c|c|c|c|}
\hline \multirow[t]{6}{*}{8} & 1 & Mollusca & $\begin{array}{l}\text { Gastro- } \\
\text { poda }\end{array}$ & $\begin{array}{l}\text { Vetigastro- } \\
\text { poda }\end{array}$ & Ovinotidae & Ovinotis & Ovinotis ovina & 3 & 0,01538 & $-4,1747$ & $-0,0642258$ & $-0,0642258$ \\
\hline & 2 & Molusca & Bivalvia & $\begin{array}{l}\text { Hipposngia- } \\
\text { dae }\end{array}$ & Corbiludae & Polymesoda & $\begin{array}{l}\text { Polymesoda } \\
\text { expansa }\end{array}$ & \begin{tabular}{|l|l|l|l}
1 \\
\end{tabular} & 0,00513 & $-5,2726$ & $-0,0270392$ & $-0,0270392$ \\
\hline & 3 & Porifera & $\begin{array}{l}\text { Gastro- } \\
\text { poda }\end{array}$ & $\begin{array}{l}\text { Caenogastro } \\
\text {-poda }\end{array}$ & Lymnaeinae & Lymneas & $\begin{array}{l}\text { Lymneas } \\
\text { stagnalis }\end{array}$ & 1 & 0,00513 & $-5,2726$ & $-0,0270392$ & $-0,0270392$ \\
\hline & 4 & Mollusca & $\begin{array}{l}\text { Gastro- } \\
\text { poda }\end{array}$ & $\begin{array}{l}\text { Caenogastro } \\
\text {-poda }\end{array}$ & Potamididae & Terebralia & \begin{tabular}{|l} 
Terebralia \\
sulcata
\end{tabular} & 4 & 0,02051 & $-3,8868$ & $-0,0797301$ & $-0,0797301$ \\
\hline & 5 & Porifera & $\begin{array}{l}\text { Gastro- } \\
\text { poda }\end{array}$ & $\begin{array}{l}\text { Neotaenioq- } \\
\text { lossa }\end{array}$ & Hydrobiidae & Pyrgulopsis & $\begin{array}{l}\text { Pyrgulopsis } \\
\text { nevadensis }\end{array}$ & 6 & 0,03077 & $-3,4812$ & $-0,1071142$ & $-0,1071142$ \\
\hline & 6 & Mollusca & Bivalvia & Veneroida & veneridae & meretrix & $\begin{array}{l}\text { Meretrix } \\
\text { meretrik }\end{array}$ & 20 & 0,10256 & $-2,2773$ & $-0,2335692$ & $-0,2335692$ \\
\hline \multirow[t]{6}{*}{9} & 1 & Mollusca & Bivalvia & Arcida & Arcidae & Arcoida & Barbatia sp. & 3 & 0,01538 & $-4,1747$ & $-0,0642258$ & $-0,0642258$ \\
\hline & 2 & Mollusca & $\begin{array}{l}\text { Gastro- } \\
\text { poda }\end{array}$ & Naticoidea & Naticidae & Arcoida & Natica sp. & 2 & 0,01026 & $-4,5795$ & $-0,0469692$ & $-0,0469692$ \\
\hline & 3 & Mollusca & $\begin{array}{l}\text { Gastro- } \\
\text { poda }\end{array}$ & $\begin{array}{l}\text { Neriti- } \\
\text { morpha }\end{array}$ & Neritoidea & Neritimorpha & Nerita $\mathrm{sp}$. & 2 & 0,01026 & $-4,5795$ & $-0,0469692$ & $-0,0469692$ \\
\hline & 4 & \begin{tabular}{|l|} 
Arthro- \\
poda
\end{tabular} & $\begin{array}{l}\text { Crusta- } \\
\text { ceae }\end{array}$ & Decapoda & Portunidae & Decapoda & Scylla sp. & 1 & 0,00513 & $-5,2726$ & $-0,0270392$ & $-0,0270392$ \\
\hline & 5 & Mollusca & $\begin{array}{l}\text { Gastro- } \\
\text { poda }\end{array}$ & Trochida & Trochidae & Arcoida & Monodonta sp. & 2 & 0,01026 & $-4,5795$ & $-0,0469692$ & $-0,0469692$ \\
\hline & 6 & Mollusca & Bivalvia & Pteriida & $\begin{array}{l}\text { Isognomo- } \\
\text { nidae }\end{array}$ & Pterioida & Isognomon sp. & 2 & 0,01026 & $-4,5795$ & $-0,0469692$ & $-0,0469692$ \\
\hline \multirow[t]{4}{*}{10} & 1 & $\begin{array}{l}\text { Arthro- } \\
\text { poda }\end{array}$ & $\begin{array}{l}\text { Malaco- } \\
\text { straca }\end{array}$ & Dacapoda & fortunidae & Scylla & Scylla serrata & 2 & 0,01026 & $-4,5795$ & $-0,0469692$ & $-0,0469692$ \\
\hline & 2 & Mollusca & Bivalvia & $\begin{array}{l}\begin{array}{l}\text { Pterio- } \\
\text { morpha }\end{array} \\
\end{array}$ & Arcidae & Anadara & $\begin{array}{l}\text { Anadara(cune } \\
\text { arca) }\end{array}$ & 13 & 0,06667 & $-2,708$ & $-0,1805333$ & $-0,1805333$ \\
\hline & 3 & Mollusca & Bivalvia & Veneroida & veneridae & Meretrix & $\begin{array}{l}\text { Meretrix } \\
\text { meretrik }\end{array}$ & 11 & 0,05641 & $-2,8751$ & $-0,1621851$ & $-0,1621851$ \\
\hline & \multicolumn{7}{|c|}{ Total } & 195 & 1 & -244153 & $-21272,933$ & $-21272,933$ \\
\hline \multicolumn{13}{|c|}{ Diversity index $\left(H^{\prime}\right)=-\sum$ Pi Ln Pi $=-(-21272,93251)=21272,93251$} \\
\hline
\end{tabular}

Table 7 Benthos Diversity of Non-Destructive Sampling in the Teupin Layeu Mangrove Waters of Sabang City

\begin{tabular}{|c|c|c|c|c|c|c|c|c|c|c|c|c|}
\hline \begin{tabular}{|c|} 
Po- \\
int
\end{tabular} & No & Phylum & Class & Ordo & Families & Genus & Species & $\Sigma$ & $\begin{array}{c}\mathrm{Pi} \\
(\mathrm{NI} / \mathrm{n})\end{array}$ & Ln. Pi & \begin{tabular}{|l|l|} 
i & Pi.Ln.PI \\
\end{tabular} & $\mathrm{H}^{\prime}$ \\
\hline \multirow[t]{3}{*}{1} & 1 & Arthropoda & Malacostraca & Decapoda & Portunidae & Scylla & Scylla olivacea & 1 & 0,00885 & -5 & $-0,041835$ & 0,0418348 \\
\hline & 2 & Mollusca & Gastropoda & Mesogastropoda & Littorinidae & Littoraria & Littoraria scabra & 2 & 0,0177 & $-4,034$ & $4-0,071402$ & 0,0714025 \\
\hline & 3 & Mollusca & Gastropoda & Cycloneritida & Neritidae & Nerita & Nerita lineata & 2 & 0,0177 & $-4,034$ & $4-0,071402$ & 0,0714025 \\
\hline \multirow[t]{6}{*}{2} & 1 & Mollusca & Gastropoda & Neritimorpha & Neritoidea & Neritimorpha & Nerita sp. & 1 & 0,00885 & -5 & $-0,041835$ & 0,0418348 \\
\hline & 2 & Mollusca & Gastropoda & Potamididae & $\begin{array}{l}\text { Caeno- } \\
\text { gastropoda }\end{array}$ & Telescopium & $\begin{array}{l}\text { Telescopium } \\
\text { telescopium }\end{array}$ & 2 & 0,0177 & $-4,034$ & $4-0,071402$ & 0,0714025 \\
\hline & 3 & Mollusca & Gastropoda & Neritimorpha & Neritoidea & Neritimorpha & Nerita sp. & 3 & 0,02655 & $-3,629$ & $-0,096339$ & 0,0963388 \\
\hline & 4 & Arthropoda & Crustaceae & Decapoda & Portunidae & Decapoda & Scylla sp. & 2 & 0,0177 & $-4,034$ & $4-0,071402$ & 0,0714025 \\
\hline & 5 & Mollusca & Gastropoda & Littorinidae & Sorbeoconcha & Littorina & Littorina scabra & 2 & 0,0177 & $-4,034$ & $4-0,071402$ & 0,0714025 \\
\hline & 6 & Mollusca & Bivalvia & Pteriida & $\begin{array}{l}\text { Isognomo- } \\
\text { nidae }\end{array}$ & Pterioida & Isognomon sp. & 4 & 0,0354 & $-3,341$ & $1-0,118269$ & 0,1182687 \\
\hline \multirow[t]{4}{*}{3} & 1 & Mollusca & Gastropoda & Vetigastropoda & Ovinotidae & Haliotis & Haliotis ovina & 2 & 0,0177 & $-4,034$ & $4-0,071402$ & 0,0714025 \\
\hline & 2 & Mollusca & Gastropoda & Caenogastropoda & Cerithidae & Rhinoclavis & $\begin{array}{l}\text { Rhinoclavis } \\
\text { sinensis }\end{array}$ & 2 & 0,0177 & $-4,034$ & $4-0,071402$ & 0,0714025 \\
\hline & 3 & Mollusca & Gastropoda & Neritimorpha & Neritidae & Neritina & Neritina natalensis & 3 & 0,02655 & $-3,629$ & $-0,096339$ & 0,0963388 \\
\hline & & Molusca & Gastropoda & Mesogastropoda & Littorinidae & Littoraria & Littoraria scabra & 1 & 0,00885 & -5 & $-0,041835$ & 0,0418348 \\
\hline \multirow[t]{2}{*}{4} & 1 & Arthropoda & Malacostraca & Decapoda & Portunidae & Scylla & Scylla olivacea & 1 & 0,00885 & -5 & $-0,041835$ & 0,0418348 \\
\hline & 2 & Mollusca & Gastropoda & Mesogastropoda & Littorinidae & Littoraria & Littoraria scabra & 2 & 0,0177 & $-4,03$ & $4-0,071402$ & 0,0714025 \\
\hline
\end{tabular}


Budapest International Research in Exact Sciences (BirEx) Journal

Volume 1, No 3, July 2019, Page: 106-114

e-ISSN: 2655-7827 (Online), p-ISSN: 2655-7835 (Print) www.bircu-journal.com/index.php/birex emails: birex.journal@gmail.com birex.journal.qa@gmail.com

\begin{tabular}{|c|c|c|c|c|c|c|c|c|c|c|c|c|}
\hline & 3 & Mollusca & Gastropoda & Cycloneritida & Neritidae & Nerita & Nerita lineata & 2 & 0,0177 & $-4,034$ & $4-0,071402$ & 0,0714025 \\
\hline \multirow[t]{4}{*}{5} & 1 & Mollusca & Bivalvia & Ptriomorphia & Arcoidae & Anadara & Anadara granosa & 6 & 0,0531 & $-2,936$ & $5-0,155874$ & 0,1558742 \\
\hline & 2 & Mollusca & Gastropoda & Caenogastropoda & Thiaridae & Tarebia & Tarebia granifera & 1 & 0,00885 & -5 & $-0,041835$ & 0,0418348 \\
\hline & 3 & Mollusca & Gastropoda & Vetigastropoda & Ovinotidae & Haliotis & Haliotis ovina & 1 & 0,00885 & -5 & $-0,041835$ & 0,0418348 \\
\hline & 4 & Mollusca & Gastropoda & Caenogastropoda & Hydrobiidae & Tryonia & Tryonia clathrata & 1 & 0,00885 & -5 & $-0,041835$ & 0,0418348 \\
\hline \multirow[t]{5}{*}{6} & 1 & Mollusca & Gastropoda & Caenogastropoda & Potamididae & Terebralia & Terebralia sulcata & 1 & 0,00885 & -5 & $-0,041835$ & 0,0418348 \\
\hline & 2 & Mollusca & Gastropoda & Megastropoda & Littorinidae & Littoraria & Littoraria scabra & 5 & 0,04425 & $-3,118$ & $3-0,13796$ & 0,1379597 \\
\hline & 3 & Mollusca & Gastropoda & & Thiaridae & Thiara & Thiara cancellata & 1 & 0,00885 & -5 & $-0,041835$ & 0,0418348 \\
\hline & 4 & Mollusca & Gastropoda & Littorinimorpba & Littorinidae & Littoraria & $\begin{array}{l}\text { Littoraria } \\
\text { anoulifera }\end{array}$ & 1 & 0,00885 & -5 & $-0,041835$ & 0,0418348 \\
\hline & 5 & Mollusca & Gastropoda & Caenogastropoda & Cerithidae & Rhinoclavis & $\begin{array}{l}\text { Rhinoclavis } \\
\text { sinensis }\end{array}$ & 1 & 0,00885 & -5 & $-0,041835$ & 0,0418348 \\
\hline & 6 & Mollusca & Gastropoda & & Ellobiidae & Cassidula & Casidula aurisfelis & 1 & 0,00885 & -5 & $-0,041835$ & 0,0418348 \\
\hline \multirow[t]{4}{*}{7} & 1 & Mollusca & Bivalvia & Pteriomorphia & Arcoidae & Anadara & Anadara granosa & 1 & 0,00885 & -5 & $-0,041835$ & 0,0418348 \\
\hline & 2 & Mollusca & Gastropoda & Vetigastropoda & Ovinotidae & Ovinotis & Ovinotis ovina & 1 & 0,00885 & -5 & $-0,041835$ & 0,0418348 \\
\hline & 3 & Mollusca & Gastropoda & Neotaenioqlossa & Hydrobiidae & Pyrgulopsis & $\begin{array}{l}\text { Prygulopsis } \\
\text { nevadensis }\end{array}$ & 1 & 0,00885 & -5 & $-0,041835$ & 0,0418348 \\
\hline & 4 & Mollusca & Gastropoda & Caenogastropoda & Thiaridae & Tarebia & Tarebia granifera & 1 & 0,00885 & -5 & $-0,041835$ & 0,0418348 \\
\hline & 5 & Mollusca & Gastropoda & Neritimorpha & Neritidae & Neritina & Neritina natalensis & 1 & 0,00885 & -5 & $-0,041835$ & 0,0418348 \\
\hline 8 & 1 & Mollusca & Bivalvia & Mytiloida & Mytilidae & Mytilus & Mytilus edulis & 2 & 0,0177 & $-4,034$ & $4-0,071402$ & 0,0714025 \\
\hline \multirow[t]{5}{*}{9} & 2 & Mollusca & Gastropoda & Neritimorpha & Neritoidea & Neritimorpha & alNerita sp. & 1 & 0,00885 & -5 & $-0,041835$ & 0,0418348 \\
\hline & 3 & Mollusca & Gastropoda & Potamididae & $\begin{array}{l}\begin{array}{l}\text { Caeno- } \\
\text { gastropoda }\end{array} \\
\end{array}$ & Telescopium & \begin{tabular}{|l} 
Telescopium \\
telescopium
\end{tabular} & 2 & 0,0177 & $-4,034$ & $4-0,071402$ & 0,0714025 \\
\hline & 4 & Mollusca & Gastropoda & Neritimorpha & Neritoidea & Neritimorpha & alNerita sp. & 3 & 0,02655 & $-3,629$ & $-0,096339$ & 0,0963388 \\
\hline & 5 & Arthropoda & Crustaceae & Decapoda & Portunidae & Decapoda & Scylla sp. & 2 & 0,0177 & $-4,034$ & $4-0,071402$ & 0,0714025 \\
\hline & 6 & Mollusca & Gastropoda & Littorinidae & Sorbeoconcha & Littorina & Littorina scabra & 2 & 0,0177 & $-4,034$ & $4-0,071402$ & 0,0714025 \\
\hline & 7 & Mollusca & Bivalvia & Pteriida & $\begin{array}{l}\text { Isognomo- } \\
\text { nidae }\end{array}$ & Pterioida & Isognomon sp. & 4 & 0,0354 & $-3,341$ & $1-0,118269$ & 0,1182687 \\
\hline \multirow[t]{3}{*}{10} & 1 & Mollusca & Bivalvia & Pteriomorpha & arcidae & anadara & Anadara(cunearca) & 23 & 0,20354 & $-1,592$ & $2-0,324013$ & 0,324013 \\
\hline & 2 & Mollusca & Bivalvia & Veneroida & veneridae & meretrix & Meretrix meretrik & 13 & 0,11504 & $-2,162$ & $2-0,24878$ & 0,2487797 \\
\hline & 3 & Mollusca & Bivalvia & Anysomyaria & mytilidae & perna & Perna viridis & 5 & 0,04425 & $-3,118$ & $3-0,13796$ & 0,1379597 \\
\hline & 113 & & -168 & $-3,211398$ & 3,2114 \\
\hline
\end{tabular}

Benthos diversity found in the waters of the Teupin Layeu mangrove, Sukakarya Subdistrict, Sabang City is 27 species using destructive sampling techniques and 29 species (Table 6).

Furthermore, using non-destructive sampling techniques with a total of 113 and 195 (Table 7). In general, the benthos species that dominate can be seen from the level of presence compared to other species. Benthos species that dominate from 10 observation points in the Teupin Layeu mangrove waters are (Meretrik meretrik) amounted to 44, and (Anadara cunearca) and amounted to 36.

\section{Conclusion}

Macrobenthos inventory on Rubiah Island in Sabang, Aceh with destructive sampling methods is amounted to 8 species from 5 families, while Benthos obtained from non- 
destructive sampling methods is amounted to 8 species from 6 families. The number of benthos species found on the Rubiah Island in the Sukakarya Subdistrict of Sabang City is 16 benthos species, with $\mathrm{H}^{\prime}=2.49655122$ indicating a moderate level of species diversity.

Macrobenthos inventory on the water of natural tourism area of Iboih beach Sabang. Diversity index is moderate with $\mathrm{H}^{\prime}=1.22061$, and non-destructive value $\mathrm{H}^{\prime}=1.31391$. Includes the category of moderate diversity.

In Payau waters with destructive $\mathrm{H}^{\prime}=1.74816$, nondestructive with a value of $\mathrm{H}^{\prime}=1.7104$. Has a moderate diversity index.

Macroentent inventory of the Teupin Layeu Mangrove Waters of Sabang City found 27 and 29 species of benthos. Among them (Meretrik meretrik) is amounted to 44, (Anadara cunearca) is amounted to 36 and many other species. With species diversity $\mathrm{H}^{\prime}=3,2114$ indicates a high level of species diversity.

\section{References}

Afkar, at el,. 2014. Asosiasi Makrozoobenthos Dengan Ekosistem Mangrove Di Sungai Reuleng Leupung, Kabupaten Aceh Besar. Journal of EduBio tropika. Vol.2. no.2

Ardian Irvan, et al. 2018. Struktur Komunitas Benthos Di Perairan Pantai Nipah Gampong Rabo Pulau Aceh Kabupaten Aceh Besar. Journal of Biotik.

Barus, T.A. 2004. Pengantar Limnologi Studi tentang Ekosistem Sungai dan Danau. Program Studi Biologi. Medan : Fakultas MIPA USU.

Borror, 2000, Ekologi Hewan, Yogyakarta: UGM Press.

Campbell, K. A. 2003. Biologi Jilid II Edisi Kelima. Jakarta: Erlangga.

Effendi. 2003. Telaah Kualitas Air Bagi Pengelolaan Sumber Daya dan Lingkungan Perairan. Jakarta: Erlangga.Fachrul, Ferianita Melati. 2006. Metode Sampling Bioekologi. Jakarta: Bumi Aksara

Janestia N.R, at el,. 2107. "Struktur Komunitas Makrozoobenthos di Ekosistem Mangrove Perairan Paru Keudee, Kabupaten Pidie Jaya”. Journal of Ilmiah Mahasiswa Kelautan dan Perikanan Unsyiah. Vol. 2. No. 3

Kamal Samsul, et al. 2013. Keanekargaman Jenis Burung Pada Perkebunan Kopi Di Kecamatan Bener Kelipah Kabupaten Bener Meriah Provinsi Aceh. Journal of Biotik. Vol. 1. No. 2.

M. Kasim Moosa, Djoko P. Praseno, Sukarno. 2008. Perairan Indonesia: Biologi, Budidaya, Kualitas Perairan, dan Oseanografi. Jakarta: Gramedia.

Mesrawaty Sabar. 2016. "Biodiversitas Dan Adaptasi Makrozoobentos Di Perairan Mangrove". Journal of Perikanan dan Ilmu Kelautan Universitas Khairun. Vol.4. No. 2

Oktavia,R. 2018. Inventarisasi Hewan Invertebrata Di Perairan Pasir Putih Lhok Mee Kabupaten Aceh Besar. Journal of Bionatural, Volume 5 Nomor 1: 61-72.

Purnami Tri Ajeng, et al. 2010. Study Of Bentos Community Based On Diversity And Similarity And Similarity Index In Cengklik Dam Boyolali. Journal of Ekosains, Vol. II. No. 2.

Razky Yatul Sidki, at el. 2016. "Struktur Komunitas Makrozoobentos Dibeberapa Muara Sungai Kecamatan Susoh Kabupaten Aceh Barat Daya". Journal of Ilmiah Mahasiswa Kelautan dan Perikanan Unsyiah. Vol. 1. No. 2

Schaduw Joshian N.W. 2018. "Distribusi Dan Karakteristik Perairan Ekosistem Mangrove Pulau Kecil Taman Nasional Bunaken”. Journal of Geografi Indonesia. Vol. 32. No. 1

T.A. Barus. 2004. Pengantai Limnologi Studi Tentang Ekosistem Air Daratan. Medan: USU Press. 